\title{
DISTRIBUTIONAL CHAOS AND FACTORS
}

\author{
JANA DOLEŽELOVÁ-HANTÁKOVÁ
}

\begin{abstract}
We show the existence of a dynamical system without any distributionally scrambled pair which is semiconjugated to a distributionally chaotic factor.

2000 Mathematics Subject Classification. Primary 37D45; 37B40.

Key words: Distributional chaos; semiconjugacy; chaotic factor.
\end{abstract}

\section{INTRODUCTION}

Semiconjugacy is used as a common tool for proving topological chaos or positive topological entropy. The usual technique is to find a semiconjugacy $\pi$ with a chaotic system and transfer the chaos to the extension. By continuity of $\pi$, the topological entropy of the extension is not smaller than the entropy of factor system. Unfortunately, semiconjugacy may not automatically guarantee the distributional chaos, which was introduced in [1]. Authors in [2, [3, [4] developed several techniques for proving distributional chaos via semiconjugacy, usually using a symbolic space as the factor space. Example in 2 shows the existence of distributionally chaotic factor which is semiconjugated to the system with no three-points distributionally scrambled sets. The aim of the paper is to improve this result and find a distributionally chaotic factor which has an extension without any distributionally scrambled pair.

\section{Terminology}

Let $(X, d)$ be a non-empty compact metric space. Let us denote by $(X, f)$ the topological dynamical system, where $f$ is a continuous self-map acting on $X$. We define the forward orbit of $x$, denoted by $\operatorname{Orb}_{f}^{+}(x)$ as the set $\left\{f^{n}(x): n \geq 0\right\}$. Let $(X, f)$ and $(Y, g)$ be dynamical systems on compact metric spaces. A continuous map $\pi: X \rightarrow Y$ is called a semiconjugacy between $\mathrm{f}$ and $\mathrm{g}$ if $\pi$ is surjective and $\pi \circ f=g \circ \pi$. In this case we can say that $(Y, g)$ is a factor of the system $(X, f)$ or eqivalently $(X, f)$ is an extension of the system $(Y, g)$.

Definition 1. A pair of two different points $\left(x_{1}, x_{2}\right) \in X^{2}$ is called scrambled if

$$
\liminf _{k \rightarrow \infty} d\left(f^{k}\left(x_{i}\right), f^{k}\left(x_{j}\right)\right)=0
$$

and

$$
\limsup _{k \rightarrow \infty} d\left(f^{k}\left(x_{i}\right), f^{k}\left(x_{j}\right)\right)>0 .
$$

A subset $S$ of $X$ is called scrambled if every pair of distinct points in $S$ scrambled. The system $(X, f)$ is called chaotic if there exists an uncountable scrambled set.

Definition 2. For a pair $\left(x_{1}, x_{2}\right)$ of points in $X$, define the lower distribution function generated by $f$ as

$$
\Phi_{\left(x_{1}, x_{2}\right)}(\delta)=\liminf _{m \rightarrow \infty} \frac{1}{m} \#\left\{0<k<m ; d\left(f^{k}\left(x_{1}\right), f^{k}\left(x_{2}\right)\right)<\delta\right\},
$$

and the upper distributional function as

$$
\Phi_{\left(x_{1}, x_{2}\right)}^{*}(\delta)=\limsup _{m \rightarrow \infty} \frac{1}{m} \#\left\{0<k<m ; d\left(f^{k}\left(x_{1}\right), f^{k}\left(x_{2}\right)\right)<\delta\right\},
$$

where \#A denotes the cardinality of the set $A$. A pair $\left(x_{1}, x_{2}\right) \in X^{2}$ is called distributionally scrambled of type 1 if

$$
\Phi_{\left(x_{1}, x_{2}\right)}^{*} \equiv 1 \text { and } \Phi_{\left(x_{1}, x_{2}\right)}(\delta)=0 \text {, for some } 0<\delta \leq \operatorname{diam} X,
$$

The research was supported by grant SGS/2/2013 from the Silesian University in Opava. Support of this institution is gratefully acknowledged. 
distributionally scrambled of type 2 if

$$
\Phi_{\left(x_{1}, x_{2}\right)}^{*} \equiv 1 \text { and } \Phi_{\left(x_{1}, x_{2}\right)}<\Phi_{\left(x_{1}, x_{2}\right)}^{*},
$$

distributionally scrambled of type 3 if

$$
\Phi_{\left(x_{1}, x_{2}\right)}<\Phi_{\left(x_{1}, x_{2}\right)}^{*} .
$$

The dynamical system $(X, f)$ is distributionally chaotic of type $i$ (DC $i$ for short), where $i=1,2,3$, if there is an uncountable set $S \subset X$ such that any pair of distinct points from $S$ is distributionally scrambled of type $i$.

\section{Distributional ChaOs AND FaCtors}

We will show the existence of a system without any distributionally scrambled pair which is semiconjugated to a distributionally chaotic factor. This system is three-dimensional union of countably many homocentric cylinders with unit height and converging radius. First we state the following technical lemma about rotation on circle. Let $u \in \mathbb{S}$ and $v \in \mathbb{S}$ be determined by normed angles $\phi_{u} \in I$ and $\phi_{v} \in I$. These points rotate along the circle by different angles $r_{u} \in I$, respectively $r_{v} \in I$, i.e.

$$
\begin{aligned}
\phi_{u} & \mapsto\left(\phi_{u}+r_{u}\right) \bmod 1 \\
\phi_{v} & \mapsto\left(\phi_{v}+r_{v}\right) \bmod 1 .
\end{aligned}
$$

We denote the relative angle of rotation by $\Delta r=\left|r_{u}-r_{v}\right|$ and assume that the metric on $\mathbb{S}$ is $\rho(\alpha, \beta)=$ $\min \{|\alpha-\beta|, 1-|\alpha-\beta|\}$.

Lemma 1. For every number $\delta>0$ and every integer $p>\frac{2}{\Delta r}$, the following estimation holds:

$$
\frac{1}{p} \#\left\{0<i<p ; \rho\left(\left(\phi_{u}+i r_{u}\right) \bmod 1,\left(\phi_{v}+i r_{v}\right) \bmod 1\right)<\delta\right\}<3 \delta .
$$

Proof. Because $\rho\left(\left(\phi_{u}+i r_{u}\right) \bmod 1,\left(\phi_{v}+i r_{v}\right) \bmod 1\right)=\rho\left(\phi_{u},\left(\phi_{v}+i \Delta r\right) \bmod 1\right)$, it is sufficinet to show

$$
\frac{1}{p} \#\left\{0<i<p ; \rho\left(\phi_{u},\left(\phi_{v}+i \Delta r\right) \bmod 1\right)<\delta\right\}<3 \delta .
$$

The expression $[p \cdot \Delta r]$ determines the number of turns that the point $v$ makes along the circle by rotation through the angle $\Delta r$ after $p$ iterations, where $[x]$ denotes the integer part of $x$. The maximal number of iterations $i$ during one turn, for which $\rho\left(\phi_{u},\left(\phi_{v}+i \Delta r\right) \bmod 1\right)<\delta$, is $\frac{2 \delta}{\Delta r}$. It follows

$$
\frac{1}{p} \#\left\{0<i<p ; \rho\left(\phi_{u},\left(\phi_{v}+i \Delta r\right) \bmod 1\right)<\delta\right\}<\frac{1}{p}\left([p \cdot \Delta r] \frac{2 \delta}{\Delta r}+\frac{2 \delta}{\Delta r}\right)<2 \delta+\frac{2 \delta}{p \Delta r} .
$$

Because $p>\frac{2}{\Delta r}$, we can estimate the second term by $\delta$, i.e. $\frac{2 \delta}{p \Delta r}<\delta$.

Theorem 1. There exists a DC1 dynamical system $(Y, f)$ which is semiconjugated to an extension $(X, F)$ which possess no distributionally scrambled pair (of type 1 or 2).

Proof. The space $X$ is defined

$$
X=\left(\left\{\left[\left(2-\frac{1}{k}\right) \cos 2 \pi \phi,\left(2-\frac{1}{k}\right) \sin 2 \pi \phi\right]: k \in \mathbb{N}, \phi \in I\right\} \bigcup\{[2 \cos 2 \pi \phi, 2 \sin 2 \pi \phi]: \phi \in I\}\right) \times I,
$$

where $I$ is the unit interval. Each point $u=\left[r_{u} \cos 2 \pi \phi_{u}, r_{u} \sin 2 \pi \phi_{u}, z_{u}\right]$ in $\mathrm{X}$ is determined by its angle $\phi_{u} \in I$, radius $r_{u} \in\left\{2-\frac{1}{k}: k \in \mathbb{N}\right\} \cup\{2\}$ and height $z_{u} \in I$.

The space is endowed with max-metric

$$
d(u, v)=\max \left\{\left|r_{u}-r_{v}\right|,\left|z_{u}-z_{v}\right|, \rho\left(\phi_{u}, \phi_{v}\right)\right\},
$$

where $\rho\left(\phi_{u}, \phi_{v}\right)=\min \left\{\left|\phi_{u}-\phi_{v}\right|, 1-\left|\phi_{u}-\phi_{v}\right|\right\}$. We define the mapping $F: X \rightarrow X$ as identity on the limit cylinder,

$$
[2 \cos 2 \pi \phi, 2 \sin 2 \pi \phi, z] \mapsto[2 \cos 2 \pi \phi, 2 \sin 2 \pi \phi, z],
$$

and as a composition of rotation and continuous mapping $g$ on inner cylinders,

$\left[\left(2-\frac{1}{k}\right) \cos 2 \pi \phi,\left(2-\frac{1}{k}\right) \sin 2 \pi \phi, z\right] \mapsto\left[\left(2-\frac{1}{k+1}\right) \cos 2 \pi(\phi+\Psi(k, z)),\left(2-\frac{1}{k+1}\right) \sin 2 \pi(\phi+\Psi(k, z)), g_{k}(z)\right]$. 
To define $g_{k}: I \rightarrow I$ and $\Psi: \mathbb{N} \times I \rightarrow I$, let $\left\{r_{i}\right\}_{i=1}^{\infty}=\left.\mathbb{Q}\right|_{(0,1)}$ be a sequence of all rationals in $(0,1)$, and $m_{1}<m_{2}<m_{3}<\ldots$ an increasing sequence of integers which we specify later. Then

$$
g_{k}=\left\{\begin{array}{ll}
h_{l} & \text { if } m_{3 l+1} \leq k<m_{3 l+2} \\
I d & \text { if } m_{3 l+2} \leq k<m_{3 l+3} \\
h_{l}^{-1} & \text { if } m_{3 l+3} \leq k<m_{3 l+4}
\end{array} \quad k, l \in \mathbb{N}_{0}\right.
$$

where $h_{l}: I \rightarrow I$ is a continuous strictly increasing mapping with three fixed points $0,1, r_{l}$ and

$$
\lim _{l \rightarrow \infty}\left\|h_{l}-I d\right\|=0 ; \quad h_{l}(x)<x \text { for } x \in\left(0, r_{l}\right) ; \quad h_{l}(x)>x \text { for } x \in\left(r_{l}, 1\right) .
$$

The sequence $\left\{m_{i}\right\}_{i=1}^{\infty}$ is defined in the following way:

$$
m_{3 l+2}-m_{3 l+1}=m_{3 l+4}-m_{3 l+3}=n_{l},
$$

where $n_{l}$ is integer satisfying

$$
h_{l}^{n_{l}}\left(\left[0, r_{l}-\frac{1}{l}\right]\right) \subset\left[0, \frac{1}{l}\right) \wedge h_{l}^{n_{l}}\left(\left[r_{l}+\frac{1}{l}, 1\right]\right) \subset\left(1-\frac{1}{l}, 1\right],
$$

and simultaneously $\left\{m_{i}\right\}_{i=1}^{\infty}$ can be chosen such that

$$
\begin{aligned}
m_{3 l+3}-m_{3 l+2}> & \frac{2 l}{\epsilon_{l}}, \quad \text { where } \epsilon_{l}=\min \left\{h_{l}^{n_{l}}\left(\frac{1}{l}\right), 1-h_{l}^{n_{l}}\left(1-\frac{1}{l}\right)\right\}, \\
& \lim _{l \rightarrow \infty} \frac{m_{3 l+1}}{m_{3 l+2}}=\lim _{l \rightarrow \infty} \frac{m_{3 l+3}}{m_{3 l+4}}=1, \\
\lim _{l \rightarrow \infty} \frac{m_{3 l+2}}{m_{3 l+3}} & =0 .
\end{aligned}
$$

The angle of rotation $\Psi: \mathbb{N} \times I \rightarrow I$ is defined as

$$
\Psi(k, z)= \begin{cases}z & \text { if } 1 \leq k<m_{4} \\ z / l & \text { if } m_{3 l+1} \leq k<m_{3 l+4} \quad l \in \mathbb{N} .\end{cases}
$$

The factor space $Y$ is simply $X$ with fixed $\phi=0$, i.e. for each point $y \in Y$,

$$
y=\left[2-\frac{1}{k}, 0, z\right] \quad \text { or } \quad[2,0, z], \quad k \in \mathbb{N}, z \in I .
$$

To simplify the notation, we skip the second zero coordinate and treat $Y$ as a two-dimensional space. The space $Y$ is union of converging sequence of unit fibers and the limit fiber,

$$
Y=\left\{2-\frac{1}{k}: k \in \mathbb{N}\right\} \times I \cup\{2\} \times I .
$$

Then the system $(X, F)$ is semiconjugated with skew-product map $f: Y \rightarrow Y$, which is identity on the limit fiber,

$$
[2, z] \mapsto[2, z]
$$

and which is $g_{k}$ on inner fibers,

$$
\left[2-\frac{1}{k}, z\right] \mapsto\left[2-\frac{1}{k+1}, g_{k}(z)\right], \quad k \in \mathbb{N} .
$$

I. The factor system $(Y, f)$ is $D C 1$.

We show that set $S=\{1\} \times I$ is a distributionally scrambled set, i.e. for any pair of distinct points $(u, v) \in S^{2}$

$$
\Phi_{(u, v)}^{*} \equiv 1 \text { and } \Phi_{(u, v)}(\epsilon)=0, \text { where } \epsilon<1 .
$$

Since $\left\{r_{i}\right\}_{i=1}^{\infty}$ is dense in $I$ and by (6), we can find a sequence $\left\{s_{k}\right\}_{k=1}^{\infty}$ such that $d\left(f^{i}(u), f^{i}(v)\right)<\frac{1}{s_{k}}$, for $m_{3 s_{k}+2} \leq i<m_{3 s_{k}+3}$, and therefore, by (8),$\Phi_{(u, v)}^{*} \equiv 1$. Suppose $u^{2}>v^{2}$, where $x^{2}$ denotes the second coordinate of a point $x$. We can find another subsequence $\left\{q_{k}\right\}_{k=1}^{\infty}$ such that $d\left(f^{i}(u), f^{i}([1,1])\right)<\frac{1}{q_{k}}$ and simultaneously $d\left(f^{i}(v), f^{i}([1,0])\right)<\frac{1}{q_{k}}$, for $m_{3 q_{k}+2} \leq i<m_{3 q_{k}+3}$. Since $f$ preserves the distance between the endpoints of any fiber, $d\left(f^{i}([1,1]), f^{i}([1,0])\right)=1$, for $i \geq 0$, we can conclude, by (8) , $\Phi_{(u, v)}(\epsilon)=0$, for any $\epsilon<1$. 
II. $(X, F)$ has no distributionally scrambled pair

We claim $\Phi_{(u, v)}^{*}<1$ for any pair of distinct points in $X$. Let $X_{0}$ be the limit cylinder $X_{0}=\{[2 \cos 2 \pi \phi, 2 \sin 2 \pi \phi]$ : $\phi \in I\} \times I$ and $\tilde{X}=X \backslash X_{0}$. Consider 4 possible cases:

a) $(u, v) \in \widetilde{X}$ with $z_{u}=z_{v}=z, k_{u}=k_{v}=k, \phi_{u} \neq \phi_{v}$.

The angle of rotation is the same for both $u$ and $v, \Psi\left(k_{u}, z_{u}\right)=\Psi\left(k_{v}, z_{v}\right)=\Psi(k, z)$, hence, by (4),

$$
d(F(u), F(v))=\rho\left(\phi_{u}+\Psi(k, z), \phi_{v}+\Psi(k, z)\right)=\rho\left(\phi_{u}, \phi_{v}\right)=d(u, v) .
$$

$F$ is isometric in this case and $\Phi_{(u, v)}^{*} \neq 1$.

b) $(u, v) \in \widetilde{X}$ with $z_{u} \neq z_{v}, k_{u}=k_{v}=k, \phi_{u} \neq \phi_{v}$

Without loss of generality suppose $k=1$ (otherwise consider the pre images $\left(F^{-k}(u), F^{-k}(v)\right)$ ) and let $L$ be an integer such that $\left|z_{u}-z_{v}\right|>\frac{1}{L}$. It is sufficient to show that there is $0<\delta<\frac{1}{3}$, for which

$$
\frac{1}{m_{3 l+3}-m_{3 l+2}} \#\left\{m_{3 l+2}<i<m_{3 l+3} ; d\left(F^{i}(u), F^{i}(v)\right)<\delta\right\}<3 \delta, \quad \text { for any } \quad L \leq l .
$$

Since $d$ is max-metric, it is sufficient to prove

$$
\frac{1}{m_{3 l+3}-m_{3 l+2}} \#\left\{m_{3 l+2}<i<m_{3 l+3} ; \rho\left(\phi_{F^{i}(u)}, \phi_{F^{i}(v)}\right)<\delta\right\}<3 \delta .
$$

Since $\left|h_{L}^{n_{L}}\left(z_{u}\right)-h_{L}^{n_{L}}\left(z_{v}\right)\right|>\epsilon_{L}$ (see (15) and definition of $\epsilon_{L}$ in (10)), and $\left|h_{L}^{n_{L}}\left(z_{u}\right)-h_{L}^{n_{L}}\left(z_{u}\right)\right|$ is the minimal distance between trajectories of $u$ and $v$ between times $m_{3 L+1}$ and $m_{3 L+4}$, it follows

$$
\min _{3 L+1<k \leq 3 L+4}\left|g_{k} \circ g_{k-1} \circ \ldots \circ g_{3 L+1}\left(z_{u}\right)-g_{k} \circ g_{k-1} \circ \ldots \circ g_{3 L+1}\left(z_{v}\right)\right|>\epsilon_{L} .
$$

Denote the relative angle of rotation of points with height $z_{u}$ and $z_{v}$ in the $k$-the cylinder by $\Delta \Psi_{k}\left(z_{u}, z_{v}\right)=$ $\left|\Psi\left(k, z_{u}\right)-\Psi\left(k, z_{v}\right)\right|=\frac{\left|z_{u}-z_{v}\right|}{L}$, for $m_{3 L+1} \leq k<m_{3 L+4}$. By (10),

$$
\Delta \Psi_{k}\left(g_{k} \circ g_{k-1} \circ \ldots \circ g_{3 L+1}\left(z_{u}\right), g_{k} \circ g_{k-1} \circ \ldots \circ g_{3 L+1}\left(z_{v}\right)\right)>\frac{\epsilon_{L}}{L} \text {, for } m_{3 L+1} \leq k<m_{3 L+4} \text {. }
$$

Since $m_{3 L+3}-m_{3 L+2}>\frac{2 L}{\epsilon_{L}}$, we can use Lemma 1 and conclude, for any $\delta>0$,

$$
\frac{1}{m_{3 L+3}-m_{3 L+2}} \#\left\{m_{3 L+2}<i<m_{3 L+3} ; \rho\left(\phi_{F^{i}(u)}, \phi_{F^{i}(v)}\right)<\delta\right\}<3 \delta .
$$

We obtain the result for any $l>L$ using the same argument, since for every $l>L,\left|z_{u}-z_{v}\right|>\frac{1}{l}$.

c) $(u, v) \in \widetilde{X}$ with $z_{u} \neq z_{v}, k_{u} \neq k_{v}, \phi_{u} \neq \phi_{v}$

Without loss of generality suppose $k_{u}=1$ and $k_{v}=p$. If $\left|z_{u}-z_{v}\right|>\frac{1}{L}$, then by case b)

$$
\#\left\{m_{4 L+2}+p<i<m_{4 L+3}-p ; \rho\left(\phi_{F^{i}(u)}, \phi_{F^{i}(v)}\right)<\delta\right\}<3 \delta \cdot\left(m_{3 L+3}-m_{3 L+2}\right)
$$

and hence

$$
\frac{1}{m_{3 L+3}-m_{3 L+2}} \#\left\{m_{3 L+2}<i<m_{3 L+3} ; \rho\left(\phi_{F^{i}(u)}, \phi_{F^{i}(v)}\right)<\delta\right\}<3 \delta+\frac{2 p}{m_{3 L+3}-m_{3 L+2}}<1,
$$

for sufficiently large $L$.

d) $u \in \widetilde{X}$ and $v \in X_{0}$

Since $v \in X_{0}$ is fixed and $\phi_{v}=\phi_{F(v)}$, we can find another point in $\tilde{X}, w=\left[\left(2-\frac{1}{k_{u}}\right) \cos 2 \pi \phi_{v},(2-\right.$ $\left.\left.\frac{1}{k_{u}}\right) \sin 2 \pi \phi_{v}, 0\right]$, which is also fixed under rotation. Therefore

$$
\rho\left(\phi_{F(u)}, \phi_{F(v)}\right)=\rho\left(\phi_{F(u)}, \phi_{F(w)}\right)
$$

and we can apply case b) or c) to investigate the pair $(u, w)$ instead of $(u, v)$.

Remark. Notice that the upper distributional function for the extension remains positive, $\Phi_{(u, v)}^{*}>0$, for any pair of distinct points in $S \times\{1\}$. By (9), $\Phi_{(u, v)}<\Phi_{(u, v)}^{*}$, hence the system $(X, F)$ is distributionally chaotic of type 3 . This fact implies an open question: Is there a DC3 system wich is semiconjugated to an extension without any distributionally scrambled pairs of type 3 ? 
Acknowledgment. I sincerely thank my supervisor, Professor Jaroslav Smítal, for valuable guidance. I am grateful for his constant support and help.

\section{REFERENCES}

[1] Schweizer B., Smítal J., Measures of chaos and a spectral decomposition of dynamical systems on the interval, Trans. Amer. Math. Soc. 344, (1994), 737 - 754.

[2] Oprocha P., WilczyŃski P., Distributional chaos via semiconjugacy, Nonlinearity 20, (2007), 2661-2679.

[3] Foryś M., Oprocha P., Wilczyński P., Factor maps and invariant distributional chaos, J. Differential Equations 256, (2014), 475-502.

[4] Wang L., Liao G., Chu Z., Duan X., The set of recurrent points of a continuous self-map on an interval and strong chaos, J. Appl. Math. Comput. 14, (2004), 277-288

J . Hantáková, Mathematical Institute, Silesian University, CZ-746 01 Opava, Czech Republic

E-mail address: jana.hantakova@math.slu.cz, 